\title{
The Uniqueness of Solution for a Class of Fractional Order Nonlinear Systems with $p$-Laplacian Operator
}

\author{
Jun-qi He and Xue-li Song \\ School of Environmental Science and Engineering, Chang'an University, Xian, Shaanxi 710054, China \\ Correspondence should be addressed to Jun-qi He; junqi_he@163.com
}

Received 21 March 2014; Accepted 16 April 2014; Published 6 May 2014

Academic Editor: Xinan Hao

Copyright (C) 2014 J.-q. He and X.-l. Song. This is an open access article distributed under the Creative Commons Attribution License, which permits unrestricted use, distribution, and reproduction in any medium, provided the original work is properly cited.

We are concerned with the uniqueness of solutions for a class of $p$-Laplacian fractional order nonlinear systems with nonlocal boundary conditions. Based on some properties of the $p$-Laplacian operator, the criterion of uniqueness for solutions is established.

\section{Introduction}

Fractional order differential systems arise from many branches of applied mathematics and physics, such as gas dynamics, Newtonian fluid mechanics, nuclear physics, and biological process [1-12]. In the recent years, there has a significant development in fractional calculus. For example, by using the contraction mapping principle, ur Rehman and Khan [13] established the existence and uniqueness of positive solutions for the fractional order differential equation with multipoint boundary conditions:

$$
\begin{aligned}
& D_{t}^{\alpha} y(t)=f\left(t, y(t), D_{t}^{\beta} y(t)\right), \quad t \in(0,1), \\
& y(0)=0, \quad D_{t}^{\beta} y(1)-\sum_{i=1}^{m-2} \zeta_{i} D_{t}^{\beta} y\left(\xi_{i}\right)=y_{0},
\end{aligned}
$$

where $1<\alpha \leq 2,0<\beta<1, \zeta_{i} \in[0,+\infty)$, and $0<\xi_{i}<1$, with $\sum_{i=1}^{m-2} \zeta_{i} \xi_{i}<1$. In [14], by using the fixed point theorem of mixed monotone operator, Zhang et al. studied the existence and uniqueness of positive solution for the following fractional order differential systems with multipoint boundary conditions:

$$
\begin{gathered}
-\mathscr{D}_{t}^{\alpha} x(t)=f\left(t, x(t), \mathscr{D}_{t}{ }^{\beta} x(t), y(t)\right), \\
-\mathscr{D}_{t}{ }^{\gamma} y(t)=g(t, x(t)), \quad t \in(0,1),
\end{gathered}
$$

$$
\begin{aligned}
& \mathscr{D}_{t}{ }^{\beta} x(0)=0, \quad \mathscr{D}_{t}{ }^{\mu} x(1)=\sum_{j=1}^{p-2} a_{j} \mathscr{D}_{t}{ }^{\mu} x\left(\xi_{j}\right), \\
& y(0)=0, \quad \mathscr{D}_{t}^{\nu} y(1)=\sum_{j=1}^{p-2} b_{j} \mathscr{D}_{t}^{\nu} y\left(\xi_{j}\right),
\end{aligned}
$$

where $1<\gamma<\alpha \leq 2,1<\alpha-\beta<\gamma, 0<\beta \leq \mu<1,0<\nu<1$, and $0<\xi_{1}<\xi_{2}<\cdots<\xi_{p-2}<1, a_{j}, b_{j} \in[0,+\infty)$ with $\sum_{j=1}^{p-2} a_{j} \xi_{j}^{\alpha-\mu-1}<1$ and $\sum_{j=1}^{p-2} b_{j} \xi_{j}^{\gamma-1}<1 ; \mathscr{D}_{t}$ is the standard Riemann-Liouville derivative. Some interesting results were also obtained by Zhang et al. [1, 2, 5, 7, 9], Goodrich [15-17], and Ahmad and Nieto [18].

On the other hand, the $p$-Laplacian equation

$$
\left(\varphi_{p}\left(x^{\prime}(t)\right)\right)^{\prime}=f\left(t, x(t), x^{\prime}(t)\right)
$$

where $\varphi_{p}(s)=|s|^{p-2} s, p>1$, can describe the turbulent flow in a porous medium; see [19]. Recently, by using Krasnoselskii' s fixed point theorem and the Leggett-Williams theorem, Wang et al. [20] investigated the existence of positive solutions for the nonlocal fractional order differential equation with a $p$-Laplacian operator:

$$
\begin{gathered}
\mathscr{D}_{t}^{\alpha}\left(\varphi_{p}\left(\mathscr{D}_{t}^{\beta} x\right)\right)(t)+f(t, x(t))=0, \\
x(0)=0, \quad \mathscr{D}_{t}^{\beta} x(0)=0, \quad x(1)=a x(\xi),
\end{gathered}
$$


where $0<\beta \leq 2,0<\alpha \leq 1,0 \leq a \leq 1$, and $0<$ $\xi<1$. And then, by looking for a more suitable upper and lower solution, Ren and Chen [21] established the existence of positive solutions for four points fractional order boundary value problem:

$$
\begin{array}{r}
\mathscr{D}_{t}^{\beta}\left(\varphi_{p}\left(\mathscr{D}_{t}^{\alpha} x\right)\right)(t)=f(t, x(t)), \quad t \in(0,1), \\
x(0)=0, \quad x(1)=a x(\xi), \\
\mathscr{D}_{t}^{\alpha} x(0)=0, \quad \mathscr{D}_{t}^{\alpha} x(1)=b \mathscr{D}_{t}^{\alpha} x(\eta),
\end{array}
$$

where $\mathscr{D}_{t}{ }^{\alpha}$ and $\mathscr{D}_{t}{ }^{\beta}$ are the standard Riemann-Liouville derivatives, $p$-Laplacian operator is defined as $\varphi_{p}(s)=|s|^{p-2} s$, $p>1$, and the nonlinearity $f$ may be singular at both $t=0,1$ and $x=0$.

Inspired by the above work, in this paper, we study the uniqueness of positive solutions for the following fractional order differential system with $p$-Laplacian operator:

$$
\begin{gathered}
\mathscr{D}_{t}^{\beta}\left(\varphi_{p_{1}}\left(\mathscr{D}_{t}^{\alpha} x\right)\right)(t)=\lambda f(t, y(t)), \\
\mathscr{D}_{t}^{\gamma}\left(\varphi_{p_{2}}\left(\mathscr{D}_{t}^{\delta} y\right)\right)(t)=\rho g(t, x(t)), \\
x(0)=0, \quad x(1)=a x(\xi), \\
\mathscr{D}_{t}^{\alpha} x(0)=0, \quad \mathscr{D}_{t}^{\alpha} x(1)=b \mathscr{D}_{t}^{\alpha} x(\eta), \\
y(0)=0, \quad y(1)=c y(\zeta), \\
\mathscr{D}_{t}^{\delta} y(0)=0, \quad \mathscr{D}_{t}^{\delta} y(1)=d \mathscr{D}_{t}^{\delta} y(\mu),
\end{gathered}
$$

where $\mathscr{D}_{t}^{\alpha}, \mathscr{D}_{t}^{\beta}, \mathscr{D}_{t}{ }^{\gamma}$, and $\mathscr{D}_{t}^{\delta}$ are the standard RiemannLiouville derivatives with $\alpha, \beta, \gamma, \delta \in(1,2], a, b, c, d \in[0,1]$, and $\xi, \eta, \zeta, \mu \in(0,1), \lambda$ and $\rho$ are positive parameters, $p$ Laplacian operator is defined as $\varphi_{p_{1}}(s)=|s|^{p_{1}-2} s, p_{1}>1$, $\left(\varphi_{p_{1}}\right)^{-1}=\varphi_{q_{1}}, 1 / p_{1}+1 / q_{1}=1$, and $\varphi_{p_{2}}(s)=|s|^{p_{2}-2} s, p_{2}>1$, $\left(\varphi_{p_{2}}\right)^{-1}=\varphi_{q_{2}}, 1 / p_{2}+1 / q_{2}=1$. In the rest of paper, we assume that $f, g:[0,1] \times \mathbb{R} \rightarrow \mathbb{R}$ are continuous.

Normally, we cannot apply the contraction mapping principle for solving the BVP (1) like ur Rehman and Khan [13] since $p$-Laplacian operator is nonlinear. In this paper, by using a property of the $p$-Laplacian operator, we overcome this difficulty and establish the uniqueness of solution for the eigenvalue problem of the fractional differential system (6).

\section{Preliminaries and Lemmas}

We firstly list the necessary definitions from fractional calculus theory here, which can be found in [10-12].

Definition 1. Let $\beta>0$. The fractional integral operator of a function $f:(0,+\infty) \rightarrow \mathbb{R}$ is given by

$$
I^{\beta} f(t)=\frac{1}{\Gamma(\beta)} \int_{0}^{t}(t-s)^{\beta-1} f(s) d s .
$$

Definition 2. Let $\beta>0$. The Riemann-Liouville fractional derivative of a function $f:(0,+\infty) \rightarrow \mathbb{R}$ is given by

$$
\mathscr{D}_{t}^{\beta} f(t)=\frac{1}{\Gamma(n-\beta)}\left(\frac{d}{d t}\right)^{n} \int_{0}^{t}(t-s)^{n-\beta-1} f(s) d s,
$$

where $n=[\beta]+1,[\beta]$ denotes the integer part of the number $\beta$, and $\Gamma$ denotes the gamma function.

Property 1. Letting $\beta>\alpha>0$ and $f \in L^{1}(0,1)$, then

$$
\begin{gathered}
I^{\beta} I^{\alpha} f(t)=I^{\beta+\alpha} f(t), \quad \mathscr{D}_{t}{ }^{\alpha} I^{\beta} f(t)=I^{\beta-\alpha} f(t), \\
\mathscr{D}_{t}{ }^{\alpha} I^{\alpha} f(t)=f(t),
\end{gathered}
$$

(2)

$$
I^{\beta} \mathscr{D}_{t}^{\beta} f(x)=f(x)+c_{1} x^{\beta-1}+c_{2} x^{\beta-2}+\cdots+c_{n} x^{\beta-n},
$$

where $c_{i} \in \mathbb{R}(i=1,2, \ldots, n)$ and $n$ is the smallest integer greater than or equal to $\beta$.

The main results of this paper are based on the following property of $p$-Laplacian operator, which is easy to be proved.

Lemma 3. (1) If $q \geq 2$ and $|x|,|y| \leq M$, then

$$
\left|\varphi_{q}(x)-\varphi_{q}(y)\right| \leq(q-1) M^{q-2}|x-y| .
$$

(2) If $1<q<2, x y>0$, and $|x|,|y| \geq m>0$, then

$$
\left|\varphi_{q}(x)-\varphi_{q}(y)\right| \leq(q-1) m^{q-2}|x-y| .
$$

Applying Definitions 1 and 2 and Property 1, we have the following lemma.

Lemma 4. Let $y \in L^{1}[0,1], 1<\alpha, \beta \leq 2,0<\xi, \eta<1$, and $0 \leq a, b \leq 1$. The fractional order boundary value problem,

$$
\begin{gathered}
\mathscr{D}_{t}^{\beta}\left(\varphi_{p_{1}}\left(\mathscr{D}_{t}^{\alpha} x\right)\right)(t)=h(t), \quad t \in(0,1), \\
x(0)=0, \quad x(1)=a x(\xi), \\
\mathscr{D}_{t}^{\alpha} x(0)=0, \quad \mathscr{D}_{t}^{\alpha} x(1)=b \mathscr{D}_{t}^{\alpha} x(\eta),
\end{gathered}
$$

has the unique solution

$$
x(t)=\int_{0}^{1} K_{1}(t, s) \varphi_{q_{1}}\left(\int_{0}^{1} K_{2}(s, \tau) h(\tau) d \tau\right) d s,
$$

where

$$
\begin{gathered}
K_{1}(t, s)=k_{1}(t, s)+\frac{a k_{1}(\xi, s) t^{\alpha-1}}{1-a \xi^{\alpha-1}}, \\
K_{2}(t, s)=k_{2}(t, s)+\frac{b_{1} k_{2}(\eta, s) t^{\beta-1}}{1-b_{1} \eta^{\beta-1}},
\end{gathered}
$$




$$
\begin{aligned}
& k_{1}(t, s)=\left\{\begin{array}{lll}
\frac{(t(1-s))^{\alpha-1}-(t-s)^{\alpha-1}}{\Gamma(\alpha)}, & 0 \leq s \leq t \leq 1, & \text { where } \\
\frac{(t(1-s))^{\alpha-1}}{\Gamma(\alpha)}, & 0 \leq t \leq s \leq 1, & \sigma_{1}(s)=\frac{b_{1} k_{2}(\eta, s)}{1-b_{1} \eta^{\beta-1}},
\end{array}\right. \\
& \left\{\frac{(t(1-s))^{\beta-1}-(t-s)^{\beta-1}}{\Gamma(\beta)}, \quad 0 \leq s \leq t \leq 1, \quad \sigma_{3}(s)=\frac{(1-s)^{\beta-1}}{\Gamma(\beta)}+\frac{b_{1} k_{2}(\eta, s)}{1-b_{1} \eta^{\beta-1}},\right. \\
& \sigma_{2}(s)=\frac{d_{1} k_{4}(\mu, s)}{1-d_{1} \mu^{\gamma-1}} \\
& \sigma_{4}(s)=\frac{(1-s)^{\gamma-1}}{\Gamma(\gamma)}+\frac{d_{1} k_{4}(\mu, s)}{1-d_{1} \mu^{\gamma-1}}
\end{aligned}
$$

and $b_{1}=b^{p_{1}-1}$.

Similar to (14), the fractional order boundary value problem,

$$
\begin{gathered}
\mathscr{D}_{t}^{\gamma}\left(\varphi_{p_{2}}\left(\mathscr{D}_{t}^{\delta} y\right)\right)(t)=h(t), \quad t \in(0,1), \\
y(0)=0, \quad y(1)=c y(\zeta), \\
\mathscr{D}_{t}^{\gamma} y(0)=0, \quad \mathscr{D}_{t}^{\delta} y(1)=d \mathscr{D}_{t}^{\delta} y(\mu),
\end{gathered}
$$

has unique solution

$$
y(t)=\int_{0}^{1} K_{3}(t, s) \varphi_{q_{2}}\left(\int_{0}^{1} K_{4}(s, \tau) h(\tau) d \tau\right) d s,
$$

where

$$
\begin{gathered}
K_{3}(t, s)=k_{3}(t, s)+\frac{c k_{3}(\zeta, s) t^{\delta-1}}{1-c \zeta^{\delta-1}}, \\
K_{4}(t, s)=k_{4}(t, s)+\frac{d_{1} k_{4}(\mu, s) t^{\gamma-1}}{1-d_{1} \mu^{\gamma-1}}, \\
k_{3}(t, s)= \begin{cases}\frac{(t(1-s))^{\delta-1}-(t-s)^{\delta-1}}{\Gamma(\delta)}, & 0 \leq s \leq t \leq 1, \\
\frac{(t(1-s))^{\delta-1}}{\Gamma(\delta)}, & 0 \leq t \leq s \leq 1,\end{cases} \\
k_{4}(t, s)= \begin{cases}\frac{(t(1-s))^{\gamma-1}-(t-s)^{\gamma-1}}{\Gamma(\gamma)}, & 0 \leq s \leq t \leq 1, \\
\frac{(t(1-s))^{\gamma-1}}{\Gamma(\gamma)}, & 0 \leq t \leq s \leq 1,\end{cases}
\end{gathered}
$$

and $d_{1}=d^{p_{2}-1}$.

Lemma 5. Let $1<\alpha, \beta, \gamma, \delta \leq 2,0<\xi, \zeta, \eta, \mu<1$, and $0 \leq a, b, c, d \leq 1$. The functions $K_{i}(t, s), i=1,2,3,4$, are continuous on $[0,1] \times[0,1]$ and satisfy

(i) $K_{i}(t, s) \geq 0, i=1,2,3,4$ for $t, s \in[0,1]$;

(ii) for $t, s \in[0,1]$,

$$
\begin{aligned}
& \sigma_{1}(s) t^{\beta-1} \leq K_{2}(t, s) \leq \sigma_{3}(s) t^{\beta-1}, \\
& \sigma_{2}(s) t^{\gamma-1} \leq K_{4}(t, s) \leq \sigma_{4}(s) t^{\gamma-1},
\end{aligned}
$$

(iii) For $t, s \in[0,1]$,

$$
K_{1}(t, s) \leq r_{1}(1-s)^{\alpha-1}, \quad K_{3}(t, s) \leq r_{2}(1-s)^{\delta-1},
$$

where

$$
\begin{aligned}
& r_{1}=\frac{1}{\Gamma(\alpha)}\left[1+\frac{a}{1-a \xi^{\alpha-1}}\right] \\
& r_{2}=\frac{1}{\Gamma(\delta)}\left[1+\frac{c}{1-c \xi^{\delta-1}}\right]
\end{aligned}
$$

Proof. The proof is obvious; we omit the proof.

The basic space used in this paper is $E=C([0,1] ; \mathbb{R}) \times$ $C([0,1] ; \mathbb{R})$, where $\mathbb{R}$ is a real number set. Obviously, the space $E$ is a Banach space if it is endowed with the norm as follows:

$$
\begin{gathered}
\|(u, v)\|:=\|u\|+\|v\|, \quad\|u\|=\max _{t \in[0,1]}|u(t)|, \\
\|v\|=\max _{t \in[0,1]}|v(t)|,
\end{gathered}
$$

for any $(u, v) \in E$. By Lemma $4,(x, y) \in E$ is a solution of the fractional order system (1) if and only if $(x, y) \in E$ is a solution of the integral equation

$$
\begin{array}{r}
x(t)=\lambda^{q_{1}} \int_{0}^{1} K_{1}(t, s) \varphi_{q_{1}}\left(\int_{0}^{1} K_{2}(s, \tau) f(s, y(\tau)) d \tau\right) d s \\
t \in[0,1] \\
y(t)=\rho^{q_{2}} \int_{0}^{1} K_{3}(t, s) \varphi_{q_{2}}\left(\int_{0}^{1} K_{4}(s, \tau) g(s, x(\tau)) d \tau\right) d s \\
t \in[0,1] .
\end{array}
$$

We define an operator $T: E \rightarrow E$ by

$$
T(x, y)(t)=(F(x, y), G(x, y)),
$$

where

$$
\begin{aligned}
& F(x, y) \\
& \quad=\lambda^{q_{1}} \int_{0}^{1} K_{1}(t, s) \varphi_{q_{1}}\left(\int_{0}^{1} K_{2}(s, \tau) f(s, y(\tau)) d \tau\right) d s,
\end{aligned}
$$




$$
\begin{aligned}
& G(x, y) \\
& \quad=\rho^{q_{2}} \int_{0}^{1} K_{3}(t, s) \varphi_{q_{2}}\left(\int_{0}^{1} K_{4}(s, \tau) g(s, x(\tau)) d \tau\right) d s .
\end{aligned}
$$

It is easy to see that $(x, y)$ is the solution of the boundary value problem (6) if and only if $(x, y)$ is the fixed point of $T$. As $f, g \in C([0,1] \times \mathbb{R}, \mathbb{R})$, we know that $T: E \rightarrow E$ is a continuous and compact operator.

\section{Main Results}

Now we here introduce a new concept: the $\mathscr{D}$-contraction mapping.

Definition 6. A function $\psi:(-\infty,+\infty) \rightarrow[0,+\infty)$ is called a nonlinear $\mathscr{D}$-contraction mapping if it is continuous and nondecreasing and satisfies $\psi(r) \leq r, r>0$.

Theorem 7. Suppose that $p_{1}, p_{2}>2$, if there exist nonnegative functions $a_{i}(t), i=1,2,3,4$, such that

$$
0<\int_{0}^{1} \delta_{i}(t) a_{i}(t) d t<+\infty, \quad i=1,2,3,4,
$$

and the following conditions are satisfied:

$\left(\mathrm{H}_{1}\right)$ for any $(t, w) \in(0,1) \times \mathbb{R}$,

$$
f(t, w) \geq a_{1}(t), \quad g(t, w) \geq a_{2}(t)
$$

$\left(\mathrm{H}_{2}\right)$ there exist $\mathscr{D}$-contraction mappings $\psi_{1}, \psi_{2}$ as

$$
\begin{array}{r}
|f(t, u)-f(t, v)| \leq a_{3}(t) \psi_{1}(|u-v|), \\
\text { a.e. }(t, u),(t, v) \in[0,1] \times \mathbb{R}, \\
|g(t, u)-g(t, v)| \leq a_{4}(t) \psi_{2}(|u-v|), \\
\text { a.e. }(t, u),(t, v) \in[0,1] \times \mathbb{R} .
\end{array}
$$

Then the fractional order differential system (6) has a unique solution provided that

$$
\begin{aligned}
\Lambda= & \lambda^{q_{1}}\left(q_{1}-1\right) r_{1} B\left(\alpha,(\beta-1)\left(q_{1}-2\right)+1\right) \\
& \times\left(\int_{0}^{1} \delta_{1}(\tau) a_{1}(\tau) d \tau\right)^{q_{1}-2} \int_{0}^{1} \delta_{3}(\tau) a_{3}(\tau) d \tau \\
& +\rho^{q_{2}}\left(q_{2}-1\right) r_{2} B\left(\delta,(\gamma-1)\left(q_{2}-2\right)+1\right) \\
& \times\left(\int_{0}^{1} \delta_{2}(\tau) a_{2}(\tau) d \tau\right)^{q_{2}-2} \int_{0}^{1} \delta_{4}(\tau) a_{4}(\tau) d \tau<1 .
\end{aligned}
$$

Proof. In the case $p_{1}, p_{2}>2$, we have $1<q_{1}, q_{2}<2$. Now we prove that $T$ is a contraction mapping. By (27)-(28) and Lemma 5, we have

$$
\begin{aligned}
& \int_{0}^{1} K_{2}(s, \tau) f(s, y(\tau)) d \tau \geq s^{\beta-1} \int_{0}^{1} \delta_{1}(\tau) a_{1}(\tau) d \tau, \\
& \int_{0}^{1} K_{4}(s, \tau) g(s, x(\tau)) d \tau \geq s^{\gamma-1} \int_{0}^{1} \delta_{2}(\tau) a_{2}(\tau) d \tau .
\end{aligned}
$$

By (12), (28), and (31), for any $\left(u_{1}, v_{1}\right),\left(u_{2}, v_{2}\right) \in E$ and for $t>0$, we have

$$
\begin{aligned}
& \mid \varphi_{q_{1}}\left(\int_{0}^{1} K_{2}(s, \tau) f\left(s, v_{1}(\tau)\right) d \tau\right) \\
& -\varphi_{q_{1}}\left(\int_{0}^{1} K_{2}(s, \tau) f\left(s, v_{2}(\tau)\right) d \tau\right) \mid \\
& \leq\left(q_{1}-1\right)\left(s^{\beta-1} \int_{0}^{1} \delta_{1}(\tau) a_{1}(\tau) d \tau\right)^{q_{1}-2} \\
& \quad \times \int_{0}^{1} K_{2}(s, \tau)\left|f\left(\tau, v_{1}(\tau)\right)-f\left(\tau, v_{2}(\tau)\right)\right| d \tau \\
& \leq\left(q_{1}-1\right)\left(s^{\beta-1} \int_{0}^{1} \delta_{1}(\tau) a_{1}(\tau) d \tau\right)^{q_{1}-2} \\
& \quad \times \int_{0}^{1} \delta_{3}(\tau) a_{3}(\tau) d \tau \psi_{1}\left(\left\|v_{1}-v_{2}\right\|\right) \\
& \leq\left(q_{1}-1\right) s^{(\beta-1)\left(q_{1}-2\right)}\left(\int_{0}^{1} \delta_{1}(\tau) a_{1}(\tau) d \tau\right)^{q_{1}-2} \\
& \quad \times \int_{0}^{1} \delta_{3}(\tau) a_{3}(\tau) d \tau\left\|v_{1}-v_{2}\right\| .
\end{aligned}
$$

Similarly, we also have

$$
\begin{aligned}
& \mid \varphi_{q_{2}}\left(\int_{0}^{1} K_{4}(s, \tau) g\left(s, u_{1}(\tau)\right) d \tau\right) \\
& -\varphi_{q_{2}}\left(\int_{0}^{1} K_{4}(s, \tau) g\left(s, u_{2}(\tau)\right) d \tau\right) \mid \\
& \leq\left(q_{2}-1\right) s^{(\gamma-1)\left(q_{2}-2\right)}\left(\int_{0}^{1} \delta_{2}(\tau) a_{2}(\tau) d \tau\right)^{q_{2}-2} \\
& \quad \times \int_{0}^{1} \delta_{4}(\tau) a_{4}(\tau) d \tau\left\|u_{1}-u_{2}\right\| .
\end{aligned}
$$

So it follows from (14), (17), and (31)-(32) that

$$
\begin{aligned}
& \left|F\left(u_{1}, v_{1}\right)(t)-F\left(u_{2}, v_{2}\right)(t)\right| \\
& =\mid \lambda^{q_{1}} \int_{0}^{1} K_{1}(t, s) \\
& \times\left[\varphi_{q_{1}}\left(\int_{0}^{1} K_{2}(s, \tau) f\left(s, v_{1}(\tau)\right) d \tau\right)\right. \\
& \left.\quad-\varphi_{q_{1}}\left(\int_{0}^{1} K_{2}(s, \tau) f\left(s, v_{2}(\tau)\right) d \tau\right)\right] d s \mid \\
& \leq \lambda^{q_{1}} r_{1} \int_{0}^{1}(1-s)^{\alpha-1} \\
& \times \mid \varphi_{q_{1}}\left(\int_{0}^{1} K_{2}(s, \tau) f\left(s, v_{1}(\tau)\right) d \tau\right)
\end{aligned}
$$




$$
\begin{aligned}
&-\varphi_{q_{1}}\left(\int_{0}^{1} K_{2}(s, \tau) f\left(s, v_{2}(\tau)\right) d \tau\right) \mid d s \\
& \leq \lambda^{q_{1}} r_{1}\left(q_{1}-1\right) \int_{0}^{1}(1-s)^{\alpha-1} s^{(\beta-1)\left(q_{1}-2\right)} d s \\
& \times\left(\int_{0}^{1} \delta_{1}(\tau) a_{1}(\tau) d \tau\right)^{q_{1}-2} \\
& \times \int_{0}^{1} \delta_{3}(\tau) a_{3}(\tau) d \tau\left\|v_{1}-v_{2}\right\| \\
& \leq \lambda^{q_{1}}\left(q_{1}-1\right) r_{1} B\left(\alpha,(\beta-1)\left(q_{1}-2\right)+1\right) \\
& \quad \times\left(\int_{0}^{1} \delta_{1}(\tau) a_{1}(\tau) d \tau\right)^{q_{1}-2} \\
& \times \int_{0}^{1} \delta_{3}(\tau) a_{3}(\tau) d \tau\left\|v_{1}-v_{2}\right\|, \\
&\left|G\left(u_{1}, v_{1}\right)(t)-G\left(u_{2}, v_{2}\right)(t)\right| \\
&=\mid \rho^{q_{2}} \int_{0}^{1} K_{3}(t, s) \\
& \quad \times \int_{0}^{1} \delta_{4}(\tau) a_{4}(\tau) d \tau\left\|u_{1}-u_{2}\right\| . \\
& \leq \rho^{q_{2}}\left(q_{2}-1\right) r_{2} B\left(\delta,(\gamma-1)\left(q_{2}-2\right)+1\right) \\
& \times\left(\int_{0}^{1} \delta_{2}(\tau) a_{2}(\tau) d \tau\right)^{q_{2}-2} \\
& \quad \times\left[\varphi_{q_{2}}\left(\int_{0}^{1} K_{4}(s, \tau) g\left(s, u_{1}(\tau)\right) d \tau\right)\right. \\
&\left.\quad \varphi_{q_{2}}\left(\int_{0}^{1} K_{4}(s, \tau) g\left(s, u_{2}(\tau)\right) d \tau\right)\right] d s \mid
\end{aligned}
$$

Hence

$$
\begin{aligned}
& \left|T\left(u_{1}, v_{1}\right)-T\left(u_{2}, v_{2}\right)\right| \\
& =\left|\left(F\left(u_{1}, v_{1}\right)-F\left(u_{2}, v_{2}\right), G\left(u_{1}, v_{1}\right)-G\left(u_{2}, v_{2}\right)\right)\right| \\
& \leq\left\|F\left(u_{1}, v_{1}\right)-F\left(u_{2}, v_{2}\right)\right\|+\left\|G\left(u_{1}, v_{1}\right)-G\left(u_{2}, v_{2}\right)\right\| \\
& \leq \lambda^{q_{1}}\left(q_{1}-1\right) r_{1} B\left(\alpha,(\beta-1)\left(q_{1}-2\right)+1\right) \\
& \quad \times\left(\int_{0}^{1} \delta_{1}(\tau) a_{1}(\tau) d \tau\right)^{q_{1}-2} \\
& \quad \times \int_{0}^{1} \delta_{3}(\tau) a_{3}(\tau) d \tau\left\|v_{1}-v_{2}\right\| \\
& \quad+\rho^{q_{2}}\left(q_{2}-1\right) r_{2} B\left(\delta,(\gamma-1)\left(q_{2}-2\right)+1\right) \\
& \quad \times\left(\int_{0}^{1} \delta_{2}(\tau) a_{2}(\tau) d \tau\right)^{q_{2}-2} \\
& \quad \times \int_{0}^{1} \delta_{4}(\tau) a_{4}(\tau) d \tau\left\|u_{1}-u_{2}\right\|
\end{aligned}
$$

$$
\begin{aligned}
& \leq \Lambda\left(\left\|v_{1}-v_{2}\right\|+\left\|u_{1}-u_{2}\right\|\right) \\
& =\Lambda\left\|\left(u_{1}, v_{1}\right)-\left(u_{2}, v_{2}\right)\right\|,
\end{aligned}
$$

where

$$
\begin{aligned}
\Lambda= & \lambda^{q_{1}}\left(q_{1}-1\right) r_{1} B\left(\alpha,(\beta-1)\left(q_{1}-2\right)+1\right) \\
& \times\left(\int_{0}^{1} \delta_{1}(\tau) a_{1}(\tau) d \tau\right)^{q_{1}-2} \int_{0}^{1} \delta_{3}(\tau) a_{3}(\tau) d \tau \\
& +\rho^{q_{2}}\left(q_{2}-1\right) r_{2} B\left(\delta,(\gamma-1)\left(q_{2}-2\right)+1\right) \\
& \times\left(\int_{0}^{1} \delta_{2}(\tau) a_{2}(\tau) d \tau\right)^{q_{2}-2} \int_{0}^{1} \delta_{4}(\tau) a_{4}(\tau) d \tau .
\end{aligned}
$$

Noticing that $0<\Lambda<1$, we obtain that $F: C[0,1] \rightarrow C[0,1]$ is a contraction mapping. By means of the Banach contraction mapping principle, we get that $T$ has a unique fixed point in $E$ which implies that the fractional order differential system (6) has a unique solution.

Theorem 8. Suppose that $1<p_{1}, p_{2} \leq 2$, if there exist nonnegative functions $b_{i}(t), i=1,2,3,4$, such that

$$
0<\int_{0}^{1} \delta_{i}(t) b_{i}(t) d t<+\infty, \quad i=1,2,3,4,
$$

and the following conditions are satisfied:

$\left(\mathrm{H}_{3}\right)$ for any $(t, w) \in(0,1) \times \mathbb{R}$,

$$
|f(t, w)| \leq b_{3}(t), \quad g(t, w) \leq b_{4}(t),
$$

$\left(\mathrm{H}_{4}\right)$ there exist $\mathscr{D}$-contraction mappings $\phi_{1}, \phi_{2}$ as

$$
\begin{array}{r}
|f(t, u)-f(t, v)| \leq b_{1}(t) \phi_{1}(|u-v|), \\
\text { a.e. }(t, u),(t, v) \in[0,1] \times \mathbb{R}, \\
|g(t, u)-g(t, v)| \leq b_{2}(t) \phi_{2}(|u-v|), \\
\text { a.e. }(t, u),(t, v) \in[0,1] \times \mathbb{R} .
\end{array}
$$

Then the fractional order differential system (6) has a unique solution provided that

$$
\begin{aligned}
\widetilde{\Lambda}= & \lambda^{q_{1}}\left(q_{1}-1\right) r_{1} B\left(\alpha,(\beta-1)\left(q_{1}-2\right)+1\right) \\
& \times\left(\int_{0}^{1} \delta_{3}(\tau) b_{3}(\tau) d \tau\right)^{q_{1}-2} \int_{0}^{1} \delta_{3}(\tau) b_{1}(\tau) d \tau \\
& +\rho^{q_{2}}\left(q_{2}-1\right) r_{2} B\left(\delta,(\gamma-1)\left(q_{2}-2\right)+1\right) \\
& \times\left(\int_{0}^{1} \delta_{4}(\tau) b_{4}(\tau) d \tau\right)^{q_{2}-2} \int_{0}^{1} \delta_{4}(\tau) b_{2}(\tau) d \tau<1 .
\end{aligned}
$$

Proof. In the case $1<p_{1}, p_{2} \leq 2$, we get $q_{1}, q_{2} \geq 2$; here we still prove that $T$ is a contraction mapping if the conditions 
6

Abstract and Applied Analysis

of theorem are satisfied. By (37)-(38) and Lemma 5, for any $(x, y) \in E$, we have

$$
\begin{aligned}
& \int_{0}^{1} K_{2}(s, \tau) f(s, y(\tau)) d \tau \leq s^{\beta-1} \int_{0}^{1} \delta_{3}(\tau) b_{3}(\tau) d \tau, \\
& \int_{0}^{1} K_{4}(s, \tau) g(s, x(\tau)) d \tau \leq s^{\gamma-1} \int_{0}^{1} \delta_{4}(\tau) b_{4}(\tau) d \tau .
\end{aligned}
$$

By (11), (39), and (41), for any $\left(u_{1}, v_{1}\right),\left(u_{2}, v_{2}\right) \in E$ and for $t>0$, we have

$$
\begin{aligned}
& \mid \varphi_{q_{1}}\left(\int_{0}^{1} K_{2}(s, \tau) f\left(s, v_{1}(\tau)\right) d \tau\right) \\
& -\varphi_{q_{1}}\left(\int_{0}^{1} K_{2}(s, \tau) f\left(s, v_{2}(\tau)\right) d \tau\right) \mid \\
& \leq\left(q_{1}-1\right)\left(s^{\beta-1} \int_{0}^{1} \delta_{3}(\tau) b_{3}(\tau) d \tau\right)^{q_{1}-2} \\
& \quad \times \int_{0}^{1} K_{2}(s, \tau)\left|f\left(\tau, v_{1}(\tau)\right)-f\left(\tau, v_{2}(\tau)\right)\right| d \tau \\
& \leq\left(q_{1}-1\right)\left(s^{\beta-1} \int_{0}^{1} \delta_{3}(\tau) b_{3}(\tau) d \tau\right)^{q_{1}-2} \\
& \quad \times \int_{0}^{1} \delta_{3}(\tau) b_{1}(\tau) d \tau \phi_{1}\left(\left\|v_{1}-v_{2}\right\|\right) \\
& \leq\left(q_{1}-1\right) s^{(\beta-1)\left(q_{1}-2\right)}\left(\int_{0}^{1} \delta_{3}(\tau) b_{3}(\tau) d \tau\right)^{q_{1}-2} \\
& \quad \times \int_{0}^{1} \delta_{3}(\tau) b_{1}(\tau) d \tau\left\|v_{1}-v_{2}\right\| .
\end{aligned}
$$

Similarly, we also have

$$
\begin{aligned}
& \mid \varphi_{q_{2}}\left(\int_{0}^{1} K_{4}(s, \tau) g\left(s, u_{1}(\tau)\right) d \tau\right) \\
& -\varphi_{q_{2}}\left(\int_{0}^{1} K_{4}(s, \tau) g\left(s, u_{2}(\tau)\right) d \tau\right) \mid \\
& \leq\left(q_{2}-1\right) s^{(\gamma-1)\left(q_{2}-2\right)} \\
& \quad \times\left(\int_{0}^{1} \delta_{4}(\tau) b_{4}(\tau) d \tau\right)^{q_{2}-2} \\
& \quad \times \int_{0}^{1} \delta_{4}(\tau) b_{2}(\tau) d \tau\left\|u_{1}-u_{2}\right\| .
\end{aligned}
$$

So it follows from (14), (17), and (42)-(43) that

$$
\begin{aligned}
& \left|F\left(u_{1}, v_{1}\right)(t)-F\left(u_{2}, v_{2}\right)(t)\right| \\
& =\mid \lambda^{q_{1}} \int_{0}^{1} K_{1}(t, s) \\
& \times\left[\varphi_{q_{1}}\left(\int_{0}^{1} K_{2}(s, \tau) f\left(s, v_{1}(\tau)\right) d \tau\right)\right. \\
& \left.-\varphi_{q_{1}}\left(\int_{0}^{1} K_{2}(s, \tau) f\left(s, v_{2}(\tau)\right) d \tau\right)\right] d s \mid
\end{aligned}
$$

$$
\begin{aligned}
& \leq \lambda^{q_{1}} r_{1} \int_{0}^{1}(1-s)^{\alpha-1} \\
& \times \mid \varphi_{q_{1}}\left(\int_{0}^{1} K_{2}(s, \tau) f\left(s, v_{1}(\tau)\right) d \tau\right) \\
& -\varphi_{q_{1}}\left(\int_{0}^{1} K_{2}(s, \tau) f\left(s, v_{2}(\tau)\right) d \tau\right) \mid d s \\
& \leq \lambda^{q_{1}} r_{1}\left(q_{1}-1\right) \int_{0}^{1}(1-s)^{\alpha-1} s^{(\beta-1)\left(q_{1}-2\right)} d s \\
& \times\left(\int_{0}^{1} \delta_{3}(\tau) b_{3}(\tau) d \tau\right)^{q_{1}-2} \\
& \times \int_{0}^{1} \delta_{3}(\tau) b_{1}(\tau) d \tau\left\|v_{1}-v_{2}\right\| \\
& \leq \lambda^{q_{1}}\left(q_{1}-1\right) r_{1} B\left(\alpha,(\beta-1)\left(q_{1}-2\right)+1\right) \\
& \times\left(\int_{0}^{1} \delta_{3}(\tau) b_{3}(\tau) d \tau\right)^{q_{1}-2} \\
& \times \int_{0}^{1} \delta_{3}(\tau) b_{1}(\tau) d \tau\left\|v_{1}-v_{2}\right\|, \\
& \left|G\left(u_{1}, v_{1}\right)(t)-G\left(u_{2}, v_{2}\right)(t)\right| \\
& =\mid \rho^{q_{2}} \int_{0}^{1} K_{3}(t, s) \\
& \times\left[\varphi_{q_{2}}\left(\int_{0}^{1} K_{4}(s, \tau) g\left(s, u_{1}(\tau)\right) d \tau\right)\right. \\
& \left.-\varphi_{q_{2}}\left(\int_{0}^{1} K_{4}(s, \tau) g\left(s, u_{2}(\tau)\right) d \tau\right)\right] d s \mid \\
& \leq \rho^{q_{2}}\left(q_{2}-1\right) r_{2} B\left(\delta,(\gamma-1)\left(q_{2}-2\right)+1\right) \\
& \times\left(\int_{0}^{1} \delta_{4}(\tau) b_{4}(\tau) d \tau\right)^{q_{2}-2} \\
& \times \int_{0}^{1} \delta_{4}(\tau) b_{2}(\tau) d \tau\left\|u_{1}-u_{2}\right\| .
\end{aligned}
$$

Hence

$$
\begin{aligned}
\mid T & \left(u_{1}, v_{1}\right)-T\left(u_{2}, v_{2}\right) \mid \\
= & \left|\left(F\left(u_{1}, v_{1}\right)-F\left(u_{2}, v_{2}\right), G\left(u_{1}, v_{1}\right)-G\left(u_{2}, v_{2}\right)\right)\right| \\
\leq & \left\|F\left(u_{1}, v_{1}\right)-F\left(u_{2}, v_{2}\right)\right\| \\
& +\left\|G\left(u_{1}, v_{1}\right)-G\left(u_{2}, v_{2}\right)\right\| \\
\leq & \lambda^{q_{1}}\left(q_{1}-1\right) r_{1} B\left(\alpha,(\beta-1)\left(q_{1}-2\right)+1\right) \\
& \times\left(\int_{0}^{1} \delta_{3}(\tau) b_{3}(\tau) d \tau\right)^{q_{1}-2} \\
& \times \int_{0}^{1} \delta_{3}(\tau) b_{1}(\tau) d \tau\left\|v_{1}-v_{2}\right\|
\end{aligned}
$$




$$
\begin{aligned}
& +\rho^{q_{2}}\left(q_{2}-1\right) r_{2} B\left(\delta,(\gamma-1)\left(q_{2}-2\right)+1\right) \\
& \times\left(\int_{0}^{1} \delta_{4}(\tau) b_{4}(\tau) d \tau\right)^{q_{2}-2} \\
& \times \int_{0}^{1} \delta_{4}(\tau) b_{2}(\tau) d \tau\left\|u_{1}-u_{2}\right\| \\
& \leq \widetilde{\Lambda}\left(\left\|v_{1}-v_{2}\right\|+\left\|u_{1}-u_{2}\right\|\right)=\widetilde{\Lambda}\left\|\left(u_{1}, v_{1}\right)-\left(u_{2}, v_{2}\right)\right\|,
\end{aligned}
$$

where

$$
\begin{aligned}
\widetilde{\Lambda}= & \lambda^{q_{1}}\left(q_{1}-1\right) r_{1} B\left(\alpha,(\beta-1)\left(q_{1}-2\right)+1\right) \\
& \times\left(\int_{0}^{1} \delta_{3}(\tau) b_{3}(\tau) d \tau\right)^{q_{1}-2} \int_{0}^{1} \delta_{3}(\tau) b_{1}(\tau) d \tau \\
& +\rho^{q_{2}}\left(q_{2}-1\right) r_{2} B\left(\delta,(\gamma-1)\left(q_{2}-2\right)+1\right) \\
& \times\left(\int_{0}^{1} \delta_{4}(\tau) b_{4}(\tau) d \tau\right)^{q_{2}-2} \int_{0}^{1} \delta_{4}(\tau) b_{2}(\tau) d \tau .
\end{aligned}
$$

Noticing that $0<\widetilde{\Lambda}<1$, we obtain that $F: C[0,1] \rightarrow C[0,1]$ is a contraction mapping. By means of the Banach contraction mapping principle, we get that $T$ has a unique fixed point in $E$ which implies that the fractional order differential system (6) has a unique solution.

It follows from Theorems 7 and 8 that the following corollaries for mixed cases hold.

Corollary 9. Suppose that $p_{1}>2$ and $1<p_{2} \leq 2$ if there exist nonnegative functions $a_{i}(t), i=1,2,3,4$, such that

$$
0<\int_{0}^{1} \delta_{i}(t) a_{i}(t) d t<+\infty, \quad i=1,2,3,4,
$$

and the following conditions are satisfied:

$\left(\mathrm{H}_{1}\right)$ for any $(t, w) \in(0,1) \times \mathbb{R}$,

$$
f(t, w) \geq a_{1}(t), \quad|g(t, w)| \leq a_{2}(t),
$$

$\left(\mathrm{H}_{2}\right)$ there exist $\mathscr{D}$-contraction mappings $\psi_{1}, \psi_{2}$ as

$$
\begin{array}{r}
|f(t, u)-f(t, v)| \leq a_{3}(t) \psi_{1}(|u-v|), \\
\text { a.e. }(t, u),(t, v) \in[0,1] \times \mathbb{R}, \\
|g(t, u)-g(t, v)| \leq a_{4}(t) \psi_{2}(|u-v|), \\
\text { a.e. }(t, u),(t, v) \in[0,1] \times \mathbb{R} .
\end{array}
$$

Then the fractional order differential system (6) has a unique solution provided that

$$
\begin{aligned}
\widetilde{\Lambda}_{1}= & \lambda^{q_{1}}\left(q_{1}-1\right) r_{1} B\left(\alpha,(\beta-1)\left(q_{1}-2\right)+1\right) \\
& \times\left(\int_{0}^{1} \delta_{1}(\tau) a_{1}(\tau) d \tau\right)^{q_{1}-2} \int_{0}^{1} \delta_{3}(\tau) a_{3}(\tau) d \tau \\
& +\rho^{q_{2}}\left(q_{2}-1\right) r_{2} B\left(\delta,(\gamma-1)\left(q_{2}-2\right)+1\right)
\end{aligned}
$$

$$
\begin{aligned}
& \times\left(\int_{0}^{1} \delta_{4}(\tau) a_{2}(\tau) d \tau\right)^{q_{2}-2} \\
& \times \int_{0}^{1} \delta_{4}(\tau) a_{4}(\tau) d \tau<1 .
\end{aligned}
$$

Corollary 10. Suppose that $p_{2}>2$ and $1<p_{1} \leq 2$ if there exist nonnegative functions $a_{i}(t), i=1,2,3,4$, such that

$$
0<\int_{0}^{1} \delta_{i}(t) a_{i}(t) d t<+\infty, \quad i=1,2,3,4,
$$

and the following conditions are satisfied:

$\left(\mathrm{H}_{1}\right)$ for any $(t, w) \in(0,1) \times \mathbb{R}$,

$$
|f(t, w)| \leq a_{1}(t), \quad g(t, w) \geq a_{2}(t),
$$

$\left(\mathrm{H}_{2}\right)$ there exist $\mathscr{D}$-contraction mappings $\psi_{1}, \psi_{2}$ as

$$
\begin{array}{r}
|f(t, u)-f(t, v)| \leq a_{3}(t) \psi_{1}(|u-v|), \\
\text { a.e. }(t, u),(t, v) \in[0,1] \times \mathbb{R}, \\
|g(t, u)-g(t, v)| \leq a_{4}(t) \psi_{2}(|u-v|), \\
\text { a.e. }(t, u),(t, v) \in[0,1] \times \mathbb{R} .
\end{array}
$$

Then the fractional order differential system (6) has a unique solution provided that

$$
\begin{aligned}
\widetilde{\Lambda}_{2}= & \lambda^{q_{1}}\left(q_{1}-1\right) r_{1} B\left(\alpha,(\beta-1)\left(q_{1}-2\right)+1\right) \\
& \times\left(\int_{0}^{1} \delta_{3}(\tau) a_{1}(\tau) d \tau\right)^{q_{1}-2} \int_{0}^{1} \delta_{3}(\tau) a_{3}(\tau) d \tau \\
& +\rho^{q_{2}}\left(q_{2}-1\right) r_{2} B\left(\delta,(\gamma-1)\left(q_{2}-2\right)+1\right) \\
& \times\left(\int_{0}^{1} \delta_{2}(\tau) a_{2}(\tau) d \tau\right)^{q_{2}-2} \\
& \times \int_{0}^{1} \delta_{4}(\tau) a_{4}(\tau) d \tau<1 .
\end{aligned}
$$

\section{Conflict of Interests}

The authors declare that there is no conflict of interests regarding the publication of this paper.

\section{Acknowledgment}

The authors were supported financially by Ministry of Education, State Administration of Foreign Experts "111 Project of Innovation and Intelligence Introducing Planning" (B08039).

\section{References}

[1] X. Zhang, L. Liu, and Y. Wu, "The uniqueness of positive solution for a singular fractional differential system involving derivatives," Communications in Nonlinear Science and Numerical Simulation, vol. 18, no. 6, pp. 1400-1409, 2013. 
[2] X. Zhang, L. Liu, and Y. Wu, "Multiple positive solutions of a singular fractional differential equation with negatively perturbed term," Mathematical and Computer Modelling, vol. 55, no. 3-4, pp. 1263-1274, 2012.

[3] X.-L. Ding and Y.-L. Jiang, "Waveform relaxation methods for fractional functional differential equations," Fractional Calculus and Applied Analysis, vol. 16, no. 3, pp. 573-594, 2013.

[4] E. Ahmed and H. A. El-Saka, "On fractional order models for Hepatitis C," Nonlinear Biomedical Physics, vol. 4, article 1, 2010.

[5] X. Zhang, L. Liu, and Y. Wu, "The eigenvalue problem for a singular higher order fractional differential equation involving fractional derivatives," Applied Mathematics and Computation, vol. 218, no. 17, pp. 8526-8536, 2012.

[6] A. A. M. Arafa, S. Z. Rida, and M. Khalil, "Fractional modeling dynamics of HIV and CD4 ${ }^{+}$T-cells during primary infection," Nonlinear Biomedical Physics, vol. 6, no. 1, article 1, 2012.

[7] X. Zhang, L. Liu, and Y. Wu, "Existence results for multiple positive solutions of nonlinear higher order perturbed fractional differential equations with derivatives," Applied Mathematics and Computation, vol. 219, no. 4, pp. 1420-1433, 2012.

[8] I. S. Jesus, J. A. T. MacHado, and J. B. Cunha, "Fractional electrical impedances in botanical elements," Journal of Vibration and Control, vol. 14, no. 9-10, pp. 1389-1402, 2008.

[9] X. Zhang, L. Liu, B. Wiwatanapataphee, and Y. Wu, "Positive solutions of eigenvalue problems for a class of fractional differential equations with derivatives," Abstract and Applied Analysis, vol. 2012, Article ID 512127, 16 pages, 2012.

[10] A. A. Kilbas, H. M. Srivastava, and J. J. Trujillo, Theory and Applications of Fractional Differential Equations, vol. 204 of North-Holland Mathematics Studies, Elsevier, Amsterdam, The Netherlands, 2006.

[11] K. S. Miller and B. Ross, An Introduction to the Fractional Calculus and Fractional Differential Equations, John Wiley \& Sons, New York, NY, USA, 1993.

[12] I. Podlubny, Fractional Differential Equations, vol. 198 of Mathematics in Science and Engineering, Academic Press, San Diego, Calif, USA, 1999.

[13] M. ur Rehman and R. A. Khan, "Existence and uniqueness of solutions for multi-point boundary value problems for fractional differential equations," Applied Mathematics Letters, vol. 23, no. 9, pp. 1038-1044, 2010.

[14] X. Zhang, L. Liu, Y. Wu, and Y. Lu, "The iterative solutions of nonlinear fractional differential equations," Applied Mathematics and Computation, vol. 219, no. 9, pp. 4680-4691, 2013.

[15] C. S. Goodrich, "Existence of a positive solution to systems of differential equations of fractional order," Computers \& Mathematics with Applications, vol. 62, no. 3, pp. 1251-1268, 2011.

[16] C. S. Goodrich, "Existence and uniqueness of solutions to a fractional difference equation with nonlocal conditions," Computers \& Mathematics with Applications, vol. 61, no. 2, pp. 191-202, 2011.

[17] C. S. Goodrich, "Positive solutions to boundary value problems with nonlinear boundary conditions," Nonlinear Analysis: Theory, Methods \& Applications, vol. 75, no. 1, pp. 417-432, 2012.

[18] B. Ahmad and J. J. Nieto, "Existence results for a coupled system of nonlinear fractional differential equations with threepoint boundary conditions," Computers \& Mathematics with Applications, vol. 58, no. 9, pp. 1838-1843, 2009.

[19] L. S. Leibenson, "General problem of the movement of a compressible fluid in a porous medium," Izvestiia Akademii Nauk Kirgizskoŭ SSSR, vol. 9, pp. 7-10, 1945 (Russian).
[20] J. Wang, H. Xiang, and Z. Liu, "Positive solutions for threepoint boundary value problems of nonlinear fractional differential equations with $p$-Laplacian," Far East Journal of Applied Mathematics, vol. 37, no. 1, pp. 33-47, 2009.

[21] T. Ren and X. Chen, "Positive solutions of fractional differential equation with $p$-Laplacian operator," Abstract and Applied Analysis, vol. 2013, Article ID 789836, 7 pages, 2013. 


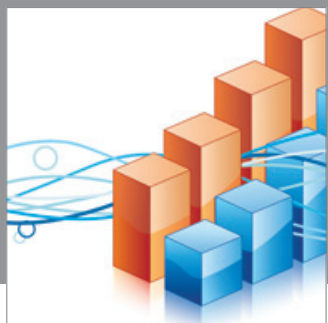

Advances in

Operations Research

mansans

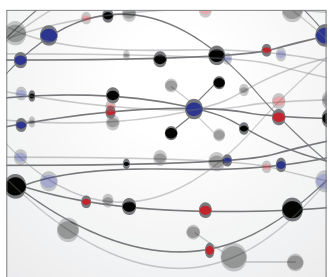

The Scientific World Journal
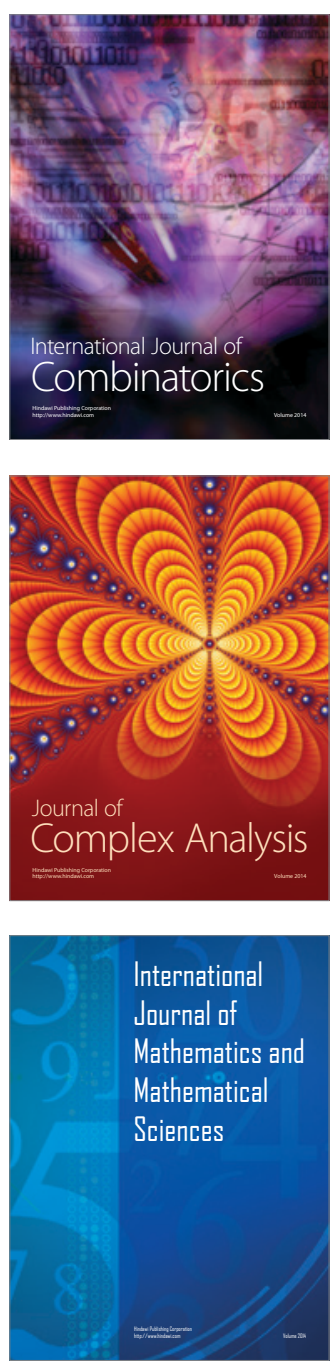
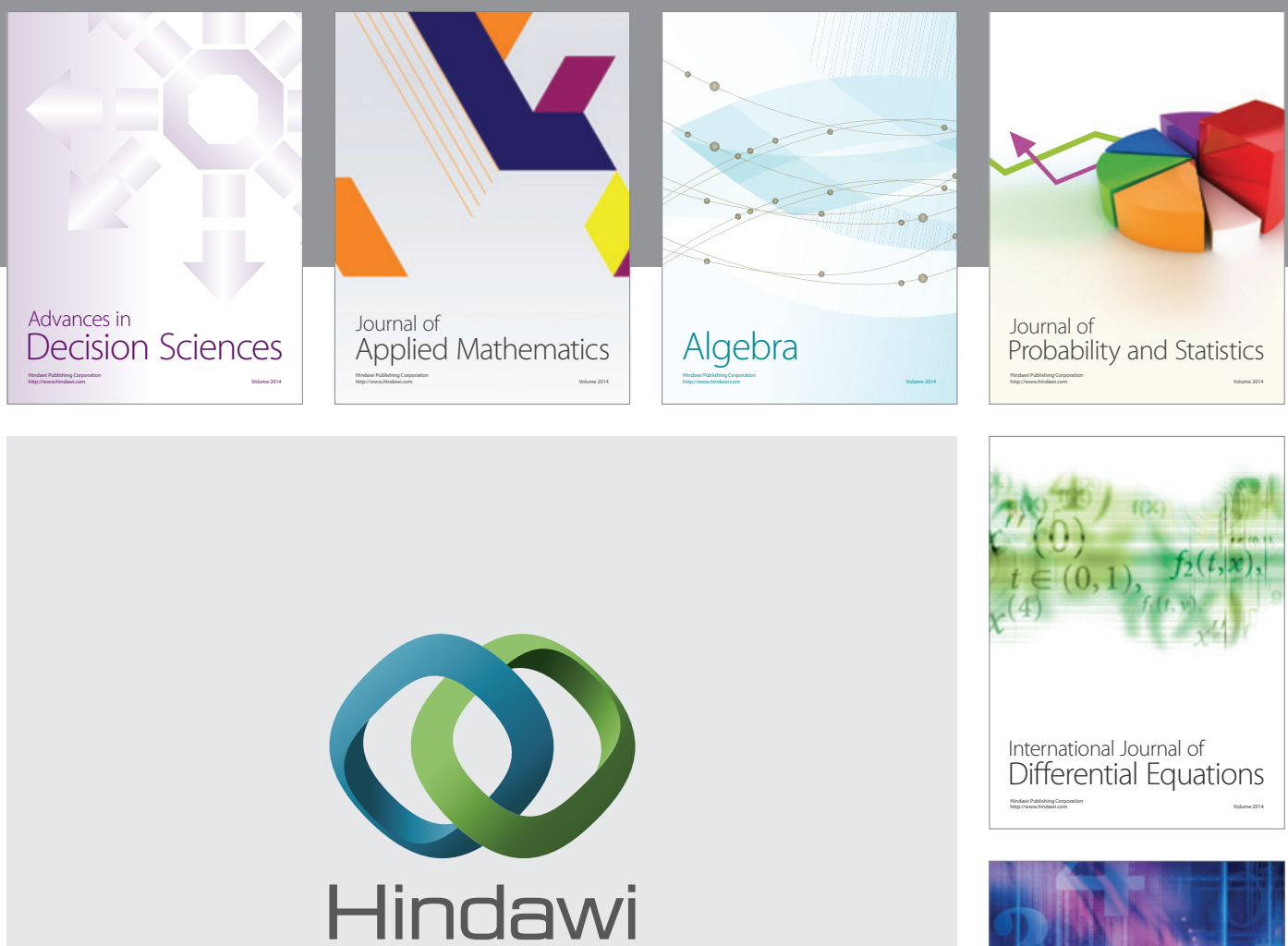

Submit your manuscripts at http://www.hindawi.com
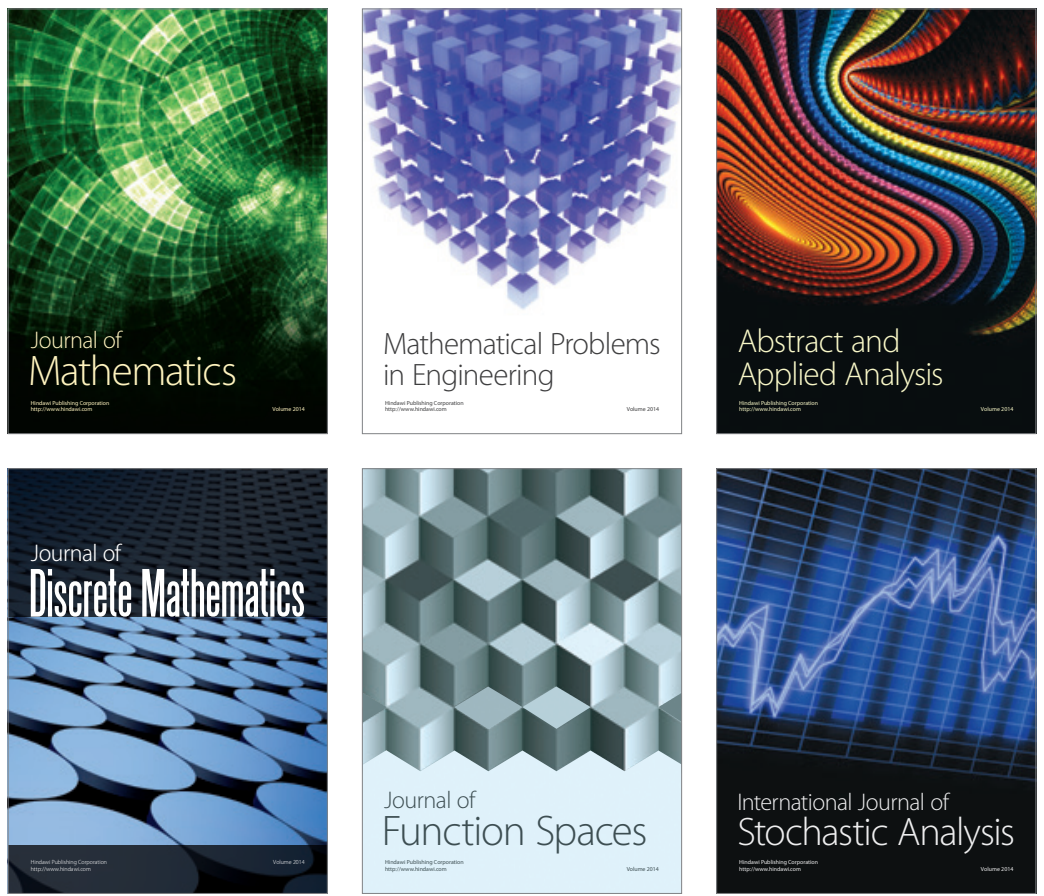

Journal of

Function Spaces

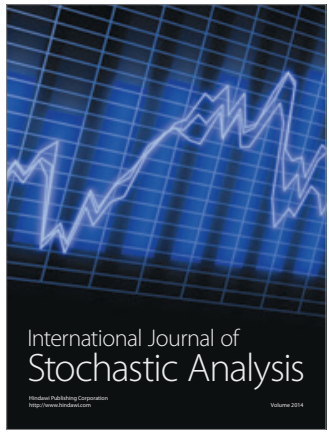

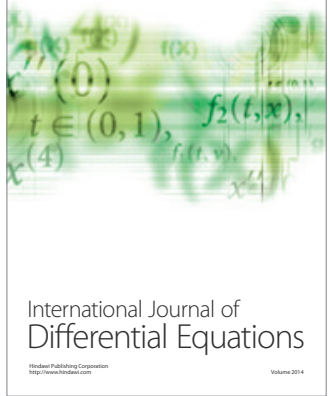
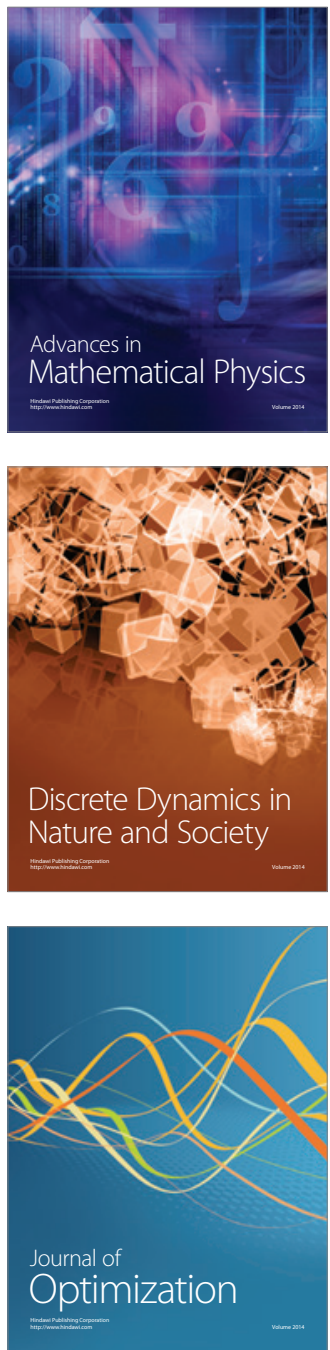